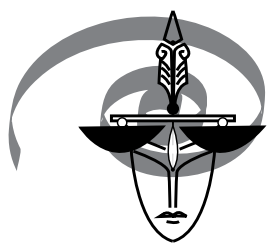

DOI: 10.1515/ep-2016-0018

\title{
Synopsis of articles from Polygraph 2015 (vol. 45) \& 2016 (vol. 46)
}

\section{- Handler M., Nelson R., \& Gougler-Floyd A. (2016), Alternative Locations for the Cardio Cuff. Is it Safe? Are the Data Similar?, Polygraph 45 (1), pp. 54-56}

The traditional blood pressure cuff used for polygraph testing has a negative impact on some examinees due to the blockage of the veins that in return affect the blood flow resulting in loss of sensation and/or skin colour changes that alarm some examinees. The article reviews alternative devices that measure blood pressure and alternative positioning of the blood pressure cuff as means of replacing the traditional cuff. The authors introduce the following alternatives:

- Finapres - a device that measures blood pressure continuously in the finger. Podlesney \& Kircher (1999) found that the Finapres offered significant correlation with the traditional cardiograph (for diastolic changes the regression coefficient mean was $\mathrm{r}=0.84$. For systolic changes, the mean was $\mathrm{r}=0.74$.) 


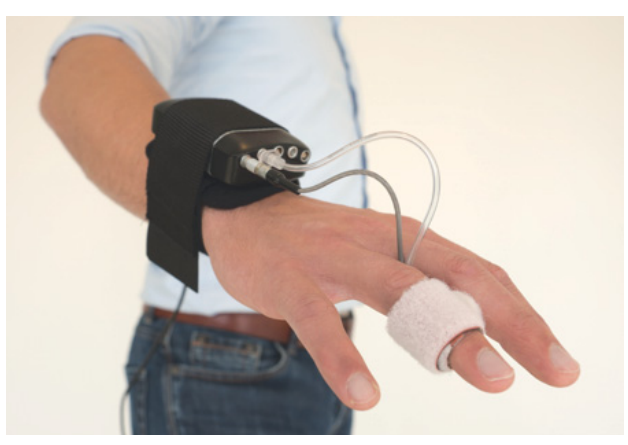

FMS | Finapres

- $\quad$ Finger cuff - Dollins \& Cestaro (1997) suggest that the finger cuff is not suitable for replacing the arm cuff due to less than sufficient correlation with the results obtained from it.

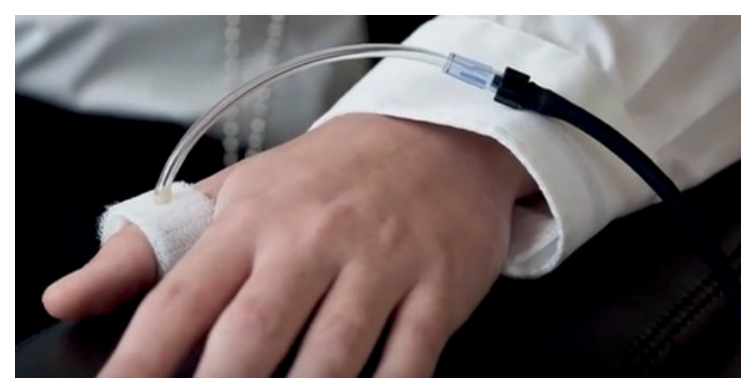

Limestone Technologies

- Lower leg or calf is an alternative location of the cuff, in which case the primary artery being monitored is the posterior tibial artery. Yet, the medical community raises concern about the location when monitoring examinees with deep venousthrombosis (DVT). Thus the selection of location should be chosen carefully to avoid blood clogs or thrombi.

- Placement of the cuff on the forearm is suggested by American Association of Critical Care Nurses. Blood pressure cuff is placed on theforearm may be better tolerated by some testsubjects, even at pressures of $80-90 \mathrm{mmHg}$.

In order to replace the arm cuff, any measuring instrument or placement method should show a high degree of correlation with the arm cuff unless replacement is verysimilar in design and use. In this case differences in tracingsare expected, which canintroduce unknown variability into polygraphscores. Initial simulations sug- 
gestthat a correlation coefficient of 0.97 will besufficient to constrain differences in scores towithin 0.5 point with both subtotal and grandtotal scores. If this correlation is achieved examiners can expect the test precision and errorrates to lie within known and established alphaboundaries.

\section{- Handler M., Honts C., Goodson W. (2015), A Literature Review of Counter- measures and Comparison Question Technique, Polygraph, 44 (2), pp. 129-137}

The article reviews history of research on the impact of countermeasures (CMs) on the comparison question technique (CQT). Before embarking on the review, the authors define $\mathrm{CMs}$ and classify various existingtypes to offer later a brief synopsis of 15 papers that investigate CMs,

The review is followed by answers to the most frequently asked questions regarding CMs.

The review gives a condensed overview of the issue and makes the article important to any polygraph practitioner in need of a quick reference guide on countermeasures.

\section{- Prado R., Grajales C., Nelson R. (2015), Laboratory Study of Directed Lie Polygraphs with Spanish Speaking Examinees, Polygraph, 44 (1), pp. 79-90}

Polygraph examinations are practiced all over the world,in probably close to a hundred different languages. We all take for granted the fact that regardless of the language spoken by the examinees; the polygraph test techniques will eventually reveal the truth, yetthis belief was not directly researched. Although the scope of the research was to examine the diagnostic accuracy of an event-specific comparison question test format in a laboratory setting. the fact that the outcome is similar to research done in other languages gives certain support to this belief.

A group of 114 randomly selected participants were divided into two equal subgroups of people innocent and guilty of stealing money from a backpack (mock crime). The participants were tested with a questionnaire that consistedof twenty two questions presented in a single sequence. The questionnaire was a single issue event-specific format that included a repetition of the two relevant questionsand two directed lie comparison questions four times, thus all together including 22 questions: 2 neutral questions inpositions 2 and 8, and repeated at positions 13 and 18; 1 sacrifice relevant question inposition 3; 2 relevant questions in positions 5 and 7 (first presentation), repeated atpositions 10 and 12 (second presentation), and 15 
and 17 (third presentation); and finally inpositions 20 and 22 (fourth presentation); 3 directed lie comparison questions in positions 4, 6, and 9, repeated at 11, 14 and 16 (secondpresentation), and again at positions 19, 21 and 23 (third presentation).

Analysis of the tests produced an unweighted accuracy of $87 \%$, with $18 \%$ inconclusiveness rate, sensitivity of $80 \%$, and specificity of $93 \%$. Reliability acc. to Kappa's statistic was 0.73 . Study results suggest accuracy greater than chance, which is consistent with other existing techniques. In addition, the results show that the effectiveness ofpolygraph examinations conducted in Spanish is similar to those conducted in English.

\section{- Nelson R. (2015), Scientific Basis for Polygraph Testing, Polygraph, 44 (1), pp. 28-61}

This paper provides all the necessary information needed for the non-expert to understand what polygraph is, while experts will find neatly and orderly structured information. The paper contains complete crucial information on the polygraph described in a crystal clear manner and supported by extensive referenceson research. Although the information in the paper is not new, the way it was put together perhaps makes itthe best paper to describe "polygraph in a nutshell" with information to be internalised by all practitioners.

The paper starts by explaining the difference between diagnostic and screening polygraph tests to continue to the description of the three phases of polygraph examination: the pre-test interview (and the differences between the free narrative, structured and semi-structured interviews, and questions review), in-test data collection (to include explanations of question structures and test formats), and test data analysis (which describes the numerical scoring). Following the description of a polygraph test, the author proceeds to the successive, deeper layer of polygraph examinations: the physiological and psychological basis of the polygraph followed by one of the most critical questions facedby examiners: the accuracy of polygraph tests. Being aware of the fact that polygraph results are probabilistic and thus not perfect, the author discusses such test pitfalls as countermeasures, "friendly examiner", and testing psychopaths. The conclusion of the paper starts from the contribution of polygraph results to professional decision making.

The author is understandably a zealous supporter of "evidence based practice", and indeed the explanations offered in the paper are evidence based and well supported. Yet, evidence based practice is a "holy" triangle (like the "fire triangle" - oxygen, heat, fuel) that integrates1) the best research evidence, with 2) the examiner's profes- 
sional expertise and discretion while considering 3) the examinee's individual conditions and personality. All the three matter for the decision making process. While the author is aware that more skilled interviewers produce better information from the examinee, he does not examine the issue more deeply, nor does he discuss contaminating factors affecting the test or the situational conditions that may affect the test outcome. Yet, judging by the author's previous publications a future "part two" of the paper is believed to cover these issues. 
